\title{
Macro-meso models for joint submitted to pyrotechnic shock
}

\author{
P.-A. Boucard ${ }^{\mathrm{a}}$, M. Dérumaux ${ }^{\mathrm{a}, *}$, P. Ladevèze ${ }^{\mathrm{a}}, \mathrm{Ph}$. Roux ${ }^{\mathrm{b}}$ \\ ${ }^{a}$ LMT Cachan, E.N.S. de Cachan - C.N.R.S. - Univ. Paris VI, 61, avenue du Président Wilson, 94235 Cachan Cedex, France \\ ${ }^{b}$ Centre National des Etudes Spatiales (C.N.E.S.), Rond point de l'Espace, 91023 Evry Cedex, France
}

\begin{abstract}
The work presented deals with the propagation of pyrotechnical shocks in complex structures and, more specifically, through joints between the stages of the Ariane 5 space launcher. As it is a failure cause for satellite equipment, the National Center for Spatial Studies (CNES) is interested in predicting the hardness levels of the shock. A computational difficulty appears near joints as phenomena deeply depend on fine geometrical and mechanical details that cannot be taken into account in the whole structure model. To carry out the calculation, a two scales strategy is used: a structure scale where the joint appears as a nonlinear interface between shells, depending on a few parameters, and a mesoscopic scale dedicated to the joint where each detail and local physical phenomena can be integrated.

Moreover, an appropriate and efficient strategy is used to take these two scales into account, identify the macro-model parameters and make it reliable. Each scale computation is described and first results are commented.
\end{abstract}

Keywords: Dynamic; Propagation; Joint; Macro-model; Multi-scale; Identification; Condensed model

\section{Industrial context}

The document reports on studies carried out in cooperation with the National Center for Spatial Studies (CNES-Evry) in the context of the "pyrotechnic shocks" interest group. The industrial goal is to better understand the shock phenomena due to the stage separation on an Ariane 5 launcher. A typical launcher structure consists of cylindrical stages coupled by bolted joints (Fig. 1).

The separation is achieved by three explosive ropes shearing the bolted joint at a speed of $7100 \mathrm{~m} / \mathrm{s}$. The crack generates a shock wave of wide frequency range and high acceleration levels. The hardness of the shock for the equipment is usually measured by shock response spectra (SRS) $[1,9]$.

To perform a correct simulation on the whole structure of the launcher, a special attention must be paid to the joint where shock reduction appears. It is the place for complex propagation and strong nonlinearities so different works are in progress at LMT Cachan to build a joint macromodel, available for standard fast dynamic finite element programs, of which parameters are adjusted from a precise propagation analysis in the bolted joint.

\footnotetext{
${ }^{*}$ Corresponding author. Tel.: +33 (1) 4740-2250; Fax: +33 (1) 4740-2785; E-mail: derumaux@lmt.ens-cachan.fr
}

\section{Computational strategy}

Two scales into the problem can be separated (Fig. 1):

- The launcher scale where the structure can be modeled by a shell assembly.

- The joint scale where nonlinearities and geometrical details need a fine description.

Our goal is to build a macro-model of the joint for the launcher scale computation, based on a few parameters with a strong mechanical meaning. Parameters have to be adjusted to fit the real behavior as well as possible. At this step, experiments would be of great help but in this case, it would be very costly to get enough data and tough to extract pertinent value from sensors. Instead of experiments, a fine 3D model of the joint, including all physical phenomena as friction, unilateral contact or plasticity is used.

Identification consists in a classical iterative method to minimize an error function. A similar approach can be found in [11]. The error function is based on the difference in the SRS responses.

\section{Efficient macro-model simulation}

The macro-model simulation consists in the calculation of the transmitted and reflected wave generated by an 


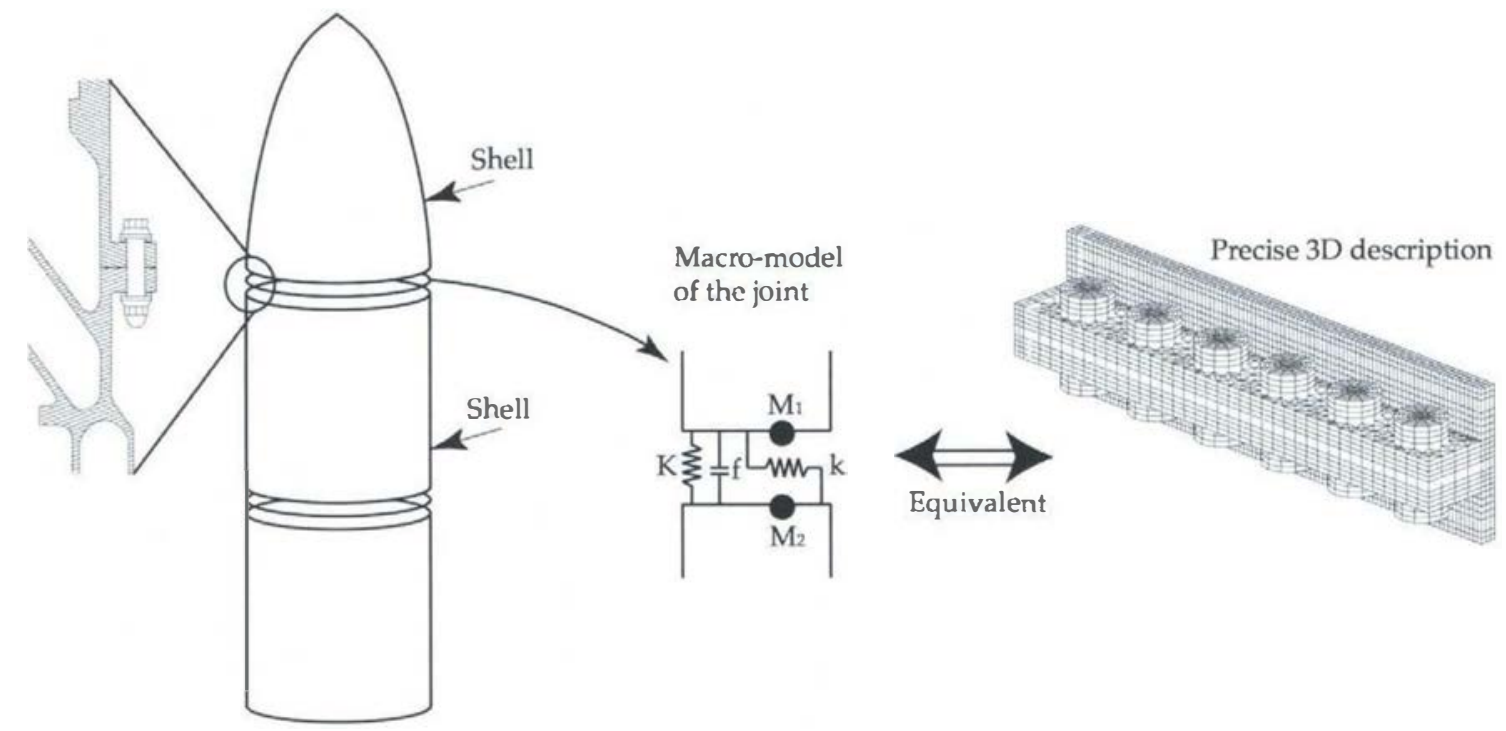

Fig. 1. Two scale strategy.
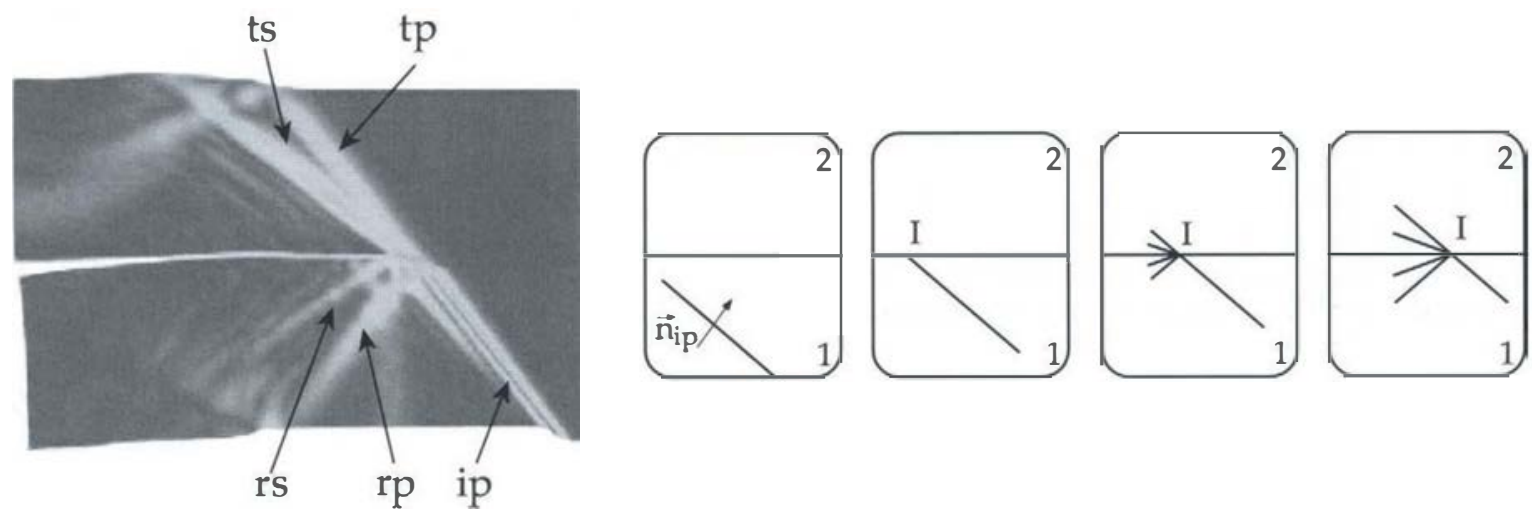

Fig. 2. Interface crossing by finite element method and analytical solution: $i=$ incident, $r=$ reflected, $t=$ transmitted, $p=$ pressure wave, and $s=$ shear wave.

incident wave (Fig. 2). A classical finite element model made of two shells jointed by an interface can get the result, as far as the model takes care of the absorption of waves on each side to avoid a non-physical multi-reflection. Then a tricky job consists in extracting the $\mathrm{P}$ (pressure) and $\mathrm{S}$ (shear) waves from the known displacement field. This is an inaccurate and lengthy way that is not adapted with an iterative identification procedure.

Finite element calculation spends all efforts in the propagation of waves in shells while the only interesting values are into the interface. As propagated solutions in shells are well known, a costly finite element method is replaced by analytical solutions.

Fig. 2 shows an incident wave split in two transmitted waves (P and $\mathrm{S})$ and two reflected waves. As the speed of the impact point $I$ is fixed by the direction $\vec{n}_{i p}$ of the incident wave, direction of those four new waves can be deducted from the incident wave. For each wave, the displacement field can be expressed by ( $c_{p}$ and $c_{s}$ are wave speeds) [5]:

- P wave: $\vec{U}_{p}(M, t)=\vec{n}_{p} \cdot f_{p}\left(\overrightarrow{O M} \cdot \vec{n}_{p}-c_{p} \cdot t\right)$

- $S$ wave: $\vec{U}_{s}(M, t)=\vec{t}_{s} \cdot f_{s}\left(\overrightarrow{O M} \cdot \vec{n}_{s}-c_{s} \cdot t\right)$

As the interface is a link between force and displacement (or speed) on each side, it is necessary to express the force on the border of the shell:

$\vec{F}(t)=\boldsymbol{\sigma}(t) \cdot \vec{y}=\mathbb{K} \cdot \boldsymbol{E}(\vec{U}(t)) \cdot \vec{y}=F \cdot \frac{\partial f}{\partial t}(t)$

Therefrom, the problem can be expressed with four time functions to determine: $f_{t p}(t), f_{t s}(t), f_{r p}(t)$ and $f_{r s}(t)$.

The resolution is processed by IATIN method where a linear global step consists in the analytical propagation problem in semi-infinite media and a nonlinear local step consists in the interface resolution problem. The robustness of the method allows strong nonlinearities as friction and unilateral contact. The simulation of a macro-model with 5 parameters as shown in Fig. 1 can be solved in less than 


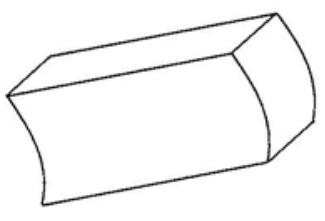

Structure

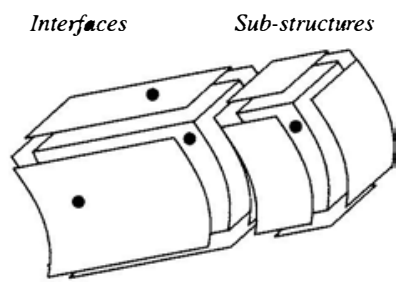

Decomposition

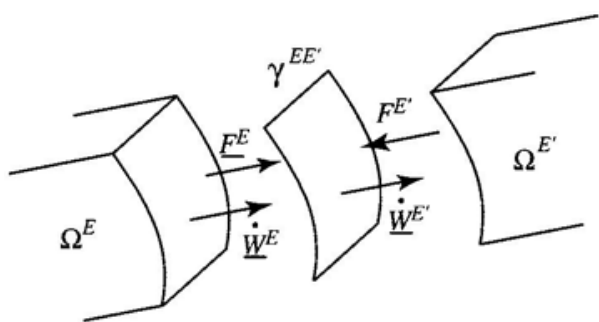

Fig. 3. Interaction of substructures and interfaces.

$2 \mathrm{~s}$ on matlab (1500 times less than FE calculation) on a standard workstation.

\section{Meso-model calculation}

The joint is studied on a small scale using a 3D mesomodel which takes the small dimensions and the material details of the actual joint into account. Appropriate numerical tools are available from previous works: the simulation is carried out with the CAST program [3,8], which uses the LATIN method [7]. The calculation is iterative and is performed globally on the whole structure and for the whole time interval. The assembly is decomposed into linear elastic substructures connected with one another through interfaces which incorporate the nonlinear phenomena (Fig. 3). This tool, which is regarded as very robust, is especially well-suited to highly nonlinear problems, such as unilateral contact or friction.

In order to solve the problem associated with the above decomposition, we use a non-incremental approach called the IATIN method first proposed by Ladevèze in 1985 (more details can be found in [6,7]). This method gives excellent results for quasi-static loading. Previous works reported computation times divided by 50 for $3 \mathrm{D}$ connection problems with many contact surfaces [2].

The LATIN method is based on three principles. The first consists of separating the difficulties in order to avoid having to deal with global and nonlinear problems simultaneously. By taking into account the mechanical properties of the equations, these can be split into two groups: the equations involving the local space variables, which may be nonlinear (the associated space will be called $\Gamma$ ) and the linear equations, which may be global in space (the associated space will be called $\mathbf{A} d$ ).

The second principle of the method involves a two-stage iteration scheme which solves each set of equations alternatively. The local stage solves the problem associated with $\Gamma$ and leads to a non-unique solution; therefore, it is necessary to add other equations $\left(E^{+}\right)$, called search direction equations. The linear global stage solves the problem associated with $\mathbf{A} d$ and leads to an ill-posed problem. It is necessary to add more search direction equations $\left(E^{-}\right)$.

The third principle of the method lies in the resolution of the global problem. A major characteristic of this computational technique is that the global operators involved at this stage remain constant throughout the iterations [8]

Fig. 4 shows a typical result of the propagation phenomena. The load is applied on the top and run at $7100 \mathrm{~m} / \mathrm{s}$. A transmitted and reflected wave can be noticed. Absorbing boundary conditions on each side avoid multiple reflection $[4,10]$.

\section{First results of identification}

Identification process is a classic iterative sheme. Several test shocks are chosen and should be representative of pyrotechnic shocks range. For each, meso-model response is calculated and gives the reference response. An iterative procedure then optimizes parameters so that the macro-model response fits the reference response.

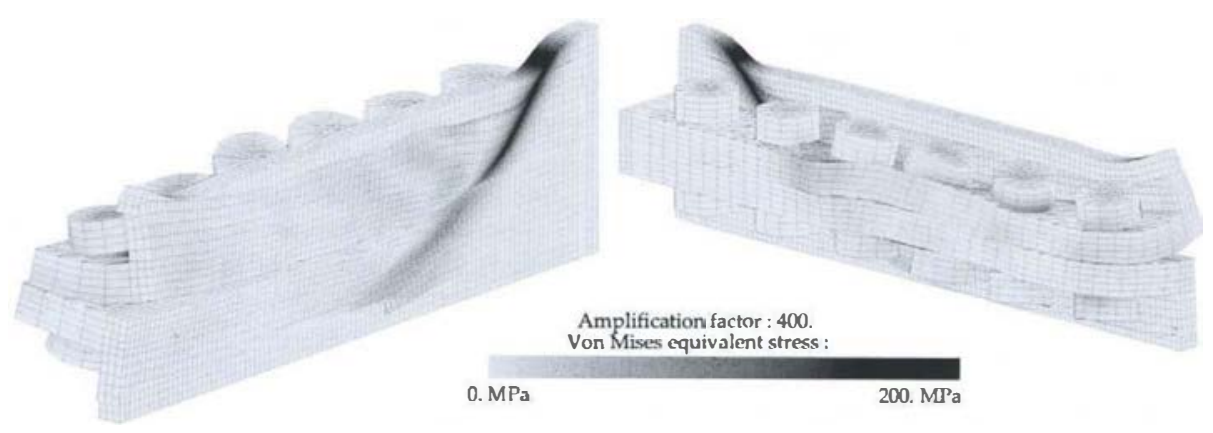

Fig. 4. Propagation phenomena in the meso-model of the joint. 

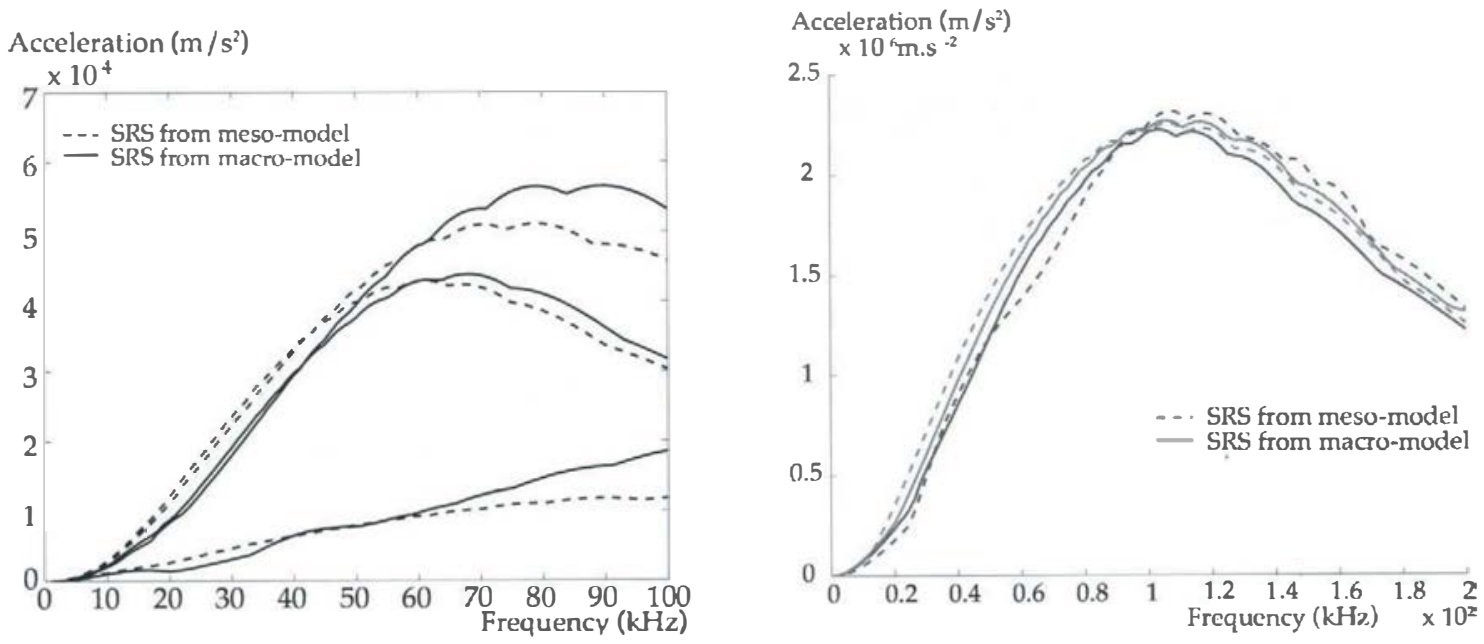

Fig. 5. Identification result of a $1 \mathrm{D}$ model (left) and 2D model (right).

Three main points will ensure the success and quality of the macro-model:

- Choice of the macro-model component: it is fundamental to include all the major physical phenomena in the macro-model; in this case, unilateral contact, friction, bolt stiffness, ect...

- Choice of the test shocks: those shocks must be representative of pyrotechnical shocks. The comparison is done by way of SRS. Giving a typical SRS, an algorithm can generate several different shocks with the same SRS.

- Choice of the gap measurement between macro-model and meso-model responses: this gap will be the error quantification to minimize. This quantity must be pertinent with regard to shocks. Once again, the maximum distance between SRS response is used.

A simplex classical algorithm that does not involve gradients is used for the minimization. This method has first been tested on a 1D optimization of macro-model. Ten test shocks are used for identification (three are plotted on Fig. 5) and a good result of $10 \%$ medium error on SRS is obtained (Fig. 5). A first 2D identification led, on one test signal, to a $12 \%$ error (Fig. 5). This encouraging result will be followed with complete $2 \mathrm{D}$ identification and testing.

\section{Conclusion}

A two-scale approach of the modeling of a joint submitted to pyrotechnic shock has been presented. Several difficulties in the calculation and identification process have led to special efficient techniques that seems to be a promising approach.

To complete FE simulation, experimental tests are currently performed by the CNES "pyrotechnical shocks" interest group. The final macro-model should easily be implemented in a classic explicit FR code.

\section{Acknowledgements}

The financial support of this work by the CNES-evry (DT99/CNES/5704) and the French Ministry of Education is gratefully acknowledged.

\section{References}

[1] Biot M. Acoustic spectrum of an elastic body submitted to shock. J Acoustical Soc Am 1934;5:206-207.

[2] Blanzé C, Champaney L, Cognard JY, Ladevèze P. A modular approach to structure assembly computations, application to contact problems. Eng Comput 1996;13(1):15-32.

[3] Champaney L, Cognard JY, Dureisseix D, Ladevèze P. Large-scale application on parallel computers of a mixed decomposition method. Comput Mech 1997;19:253-263.

[4] Engquist B, Majda A. Absorbing boundary conditions for the numerical simulation of waves. Math Comput 1977; 139:629-651.

[5] Graff KF. Wave Motion in Elastic Solids. New York: Dover, 1991.

[6] Ladevèze P. Sur une famille d'algorithmes en mécanique des structures. C R Acad Sci Paris 1985;II:41-44.

[7] Ladevèze P. Non-linear computational structural mechanics. New York, NY: Springer, 1998 (French version 1996).

[8] Ladevèze P, Lemoussu H, Boucard P. A modular approach to 3D-impact computation with frictional contact. Comput Struct 2000;78(1/3):45-52.

[9] Lalanne C. Mechanical Shocks (Vol. 2). London: Hermes Penton Science, 2002.

[10] Lysmer J, Kuhlemeyer R. Finite dynamic model for infinite media. J Eng Mech Div ASCE 1969;95-4.

[11] Wang J, Sas P. A method for identifying parameters of mechanical joints. J Appl Mech 1990;57:261-271. 